\title{
Decoherence can help quantum cryptographic security
}

\author{
Vishal Sharma, ${ }^{1}$ U. Shrikant,${ }^{2,3}$ R. Srikanth, ${ }^{2}$ and Subhashish Banerjee ${ }^{4}$ \\ ${ }^{1}$ IIT Jodhpur, Jodhpur, Rajasthan India* \\ ${ }^{2}$ Poornaprajna Institute of Scientific Research, Bangalore - 562164, India ${ }^{\dagger}$ \\ ${ }^{3}$ Graduate Studies, Manipal University, Manipal-576104 \\ ${ }^{4}$ IIT Jodhpur, Rajasthan India ${ }^{\ddagger}$
}

\begin{abstract}
In quantum key distribution, one conservatively assumes that the eavesdropper Eve is restricted only by physical laws, whereas the legitimate parties, namely the sender Alice and receiver Bob, are subject to realistic constraints, such as noise due to environment-induced decoherence. In practice, Eve too may be bound by the limits imposed by noise, which can give rise to the possibility that decoherence works to the advantage of the legitimate parties. A particular scenario of this type is one where Eve can't replace the noisy communication channel with an ideal one, but her eavesdropping channel itself remains noiseless. Here, we point out such a situation, where the security of the Ping-Pong protocol (modified to a key distribution scheme) against a noise-restricted adversary improves under a non-unital noisy channel, but deteriorates under unital channels. This highlights the surprising fact that, contrary to the conventional expectation, noise can be helpful to quantum information processing. Furthermore, we point out that the measurement outcome data in the context of the non-unital channel can't be simulated by classical noise locally added by the legitimate users.
\end{abstract}

\section{INTRODUCTION}

Cryptography helps secure information being communicated between legitimate users [1, 2] across a quantum communication channel [3-6], which may be optical, open space or satellite-based [7, 8]. Since the seminal BB84 quantum key distribution (QKD) proposal [9], the idea that unconditional security of the distributed key can be obtained by using quantum resources has been extensively studied through more detailed security analyses and newer QKD protocols, among them [10-16]. See Refs. [17] and [18] and references therein.

A variant of QKD is one involving direct communication avoiding the step of key generation [19]. These protocols may be classified as QSDC (quantum secure direct communication) [20-22] and DSQC (deterministic secure quantum communication) protocols. The difference is that, unlike DSQC protocols, QSDC protocols don't require any additional classical communication, except for checking eavesdropping. Other important cryptotasks under active investigation include quantum coin flipping [23], quantum money [24], quantum private query [25], quantum secure computation [26].

Environmental noise is ubiquitous in the real world, and is generally detrimental to quantum communication [27-30]. In quantum key distribution, it is conservative to assume that all of the noise is due to an eavesdropper Eve, who replaces the noisy (and/or lossy) channel with an ideal one [31]. Eve is assumed to be as powerful as the laws of physics would allow. This determines the largest noise level that can be tolerated.

\footnotetext{
*pg201383506@iitj.ac.in

† shrik@poornaprajna.org

‡ subhashish@iitj.ac.in
}

In reality, we may expect that Eve, too, to be restricted by the noise. Alice, Bob and Eve may be assumed to be aware of this. As the legitimate and eavesdropping channels are not identical, this scenario of noise-restricted Eve gives rise to the interesting possibility that noise may be more disadvantageous for Eve than for Alice and Bob. Here we shall present a concrete instance of such a situation. This can be trivially ensured by making the eavesdropping channel more noisy than Alice's and Bob' communication channel. A more non-trivial scenario is one where the noisy channel acts directly only on the communication channel and not on the eavesdropping channel. On the other hand, Eve is assumed to be unable to replace the noisy channel of Alice and Bob with an ideal one.

Our main result is the demonstration of a quantum key distribution (QKD) situation where non-unital noise can be beneficial to the legitimate participants in this sense, whereas unital noise is detrimental to them. This can potentially form the basis for "trusted noise", wherein Alice and Bob add noise prior to classical post-processing to improve the protocol's security or performance. Interestingly, such an application of noise for QKD has been noted earlier. In particular, in an analysis of various QKD protocols, [32] shows that they can be made more robust against channel noise by the addition of noise by Alice or Bob to the measurement data prior to key reconciliation. Refs. [33, 34] discuss adding noise to the signal to improve noise tolerance in the context of continuousvariable QKD over Gaussian channels. Interestingly, a somewhat similar favorable effect of noise on quantum information processing was noted in [35].

Secure direct communication (SDC) is a stronger form of secure communication than key distribution wherein message bits, rather than random key bits, are transmitted from sender Alice to receiver Bob. Since the proposal of the first quantum SDC protocol, namely the Ping-pong 
protocol [20], a number of other realizations of this theme have been proposed [36-43]. The Ping-Pong protocol's security, as well as its modified versions, have been extensively studied by various other authors [37, 44-56]. A comprehensive review of some of the attacks and protective measures against them are discussed by the authors of the Ping-pong protocol [57].

The original Ping-pong protocol is based on two modes: the message mode during which a bit is transmitted deterministically, and control mode, to monitor eavesdropping. This structure is necessitated by the requirement for the protocol to perform as a scheme for SDC. Here, however, we will use a simplified version of the Ping-pong protocol (though it will still be called as such), which is suitable for key distribution, but in general not for SDC. This is done by dropping the control mode, and instead using a quantum bit error rate (QBER) analysis (which involves sacrficing some otherwise secret bits) for detecting eavesdropping.

For our purpose, it will suffice to consider the depolarizing and AD (amplitude damping) channels, representative of unital channels (those that map the identity operator to itself) and non-unital channels, respectively. Furthermore, the noise acts only on the communication channel and not directly on the eavesdropping channel, so that Eve is affected only by the interaction of her probes with the noisy communication channel, rather than noise acting on her probes directly. In this scenario, the semipowerful Eve is able to deploy noiseless probes, but unable to replace Alice-Bob's noisy channel with a noiseless one.

The remaining work is divided as follows. In Section II, we briefly review the Ping-Pong protocol reformulated as a QKD (rather than SDC) scheme, and an attacking strategy on it [44]. In Section III, we introduce the noise scenario used in this work. In Sections III A and III B, we study the performance of the (modified) Ping-Pong protocol in the presence of the $\mathrm{AD}$ and depolarizing channels, respectively, pointing out the (surprisingly) beneficial aspect of the former. The question of the simulation of the measurement outcome data under a noisy channel by the resource of local classical noise applied by the legitimate users, is considered in the conclusing Section IV.

\section{EAVESDROPPING ON THE PING-PONG PROTOCOL}

First, we briefly describe the (modified) Ping-Pong key distribution protocol, based on the original secure deterministic communication protocol [20]. In what follows, we use the notation where $|0\rangle$ and $|1\rangle$ represent the two polarization states $H$ and $V$ of a single photon, respectively, whilst $|2\rangle$ represents the vacuum state.

1. Bob transmits to Alice one half (the "travel qubit") of the Bell state $\left|\psi^{+}\right\rangle=\frac{1}{\sqrt{2}}(|01\rangle+|10\rangle)$.
2. Alice encodes one bit of information by applying operation $I$ (resp., Pauli $\sigma_{Z}$ ), corresponding to the bit value $a=0$ (resp., $a=1$ ).

3. She retransmits the travel qubit back to Bob.

4. The two-qubit state now left with Bob is ideally in one the Bell states $\left|\psi^{ \pm}\right\rangle$, which is determined by Bob by a Bell-state measurement.

5. For a sufficiently large set of the (noisy) shared bits, Alice announces the encoded bit on some of the transmissions. The fraction of bits where Alice's and Bob's records differ determines the quantum bit error rate (QBER). If the QBER is below a threshold value, they proceed to distill a secret key. Else, they abort.

Wöjcik proposed an eavesdropping strategy on the original ping-pong protocol, which is now adapted for the modified Ping-pong protocol. The basic intuition of security in the Ping-pong protocol is that the travel qubit remains always in the maximally mixed state, irrespective of Alice's encoding. The subtlety of Wöjcik's attack is that by making the probe interact before and after the encoding, Eve is able to extract some information about the encoding. A brief description of the attack adapted to the above protocol is enumerated below.

1. Eve prepares two probes $x$ and $y$ in the state $|2\rangle_{x}|0\rangle_{y}$, where $|2\rangle$ is the vacuum state. Thus, the combined initial quantum state with Bob and Eve is $\left|\psi^{\text {initial }}\right\rangle=\left|\psi^{+}\right\rangle_{h t}|2\rangle_{x}|0\rangle_{y}$.

2. In the onward leg, Eve attacks the travel qubit by applying the operation $Q_{t x y}=$ $S W A P_{t x} C P B S_{t x y} H_{y}$, with CPBS being the controlled polarization beam splitter operation, given by:

$$
\left.\begin{array}{l}
|020\rangle \\
|021\rangle \\
|120\rangle \\
|121\rangle
\end{array}\right\} \stackrel{C P B S}{\longrightarrow}\left\{\begin{array}{l}
|002\rangle \\
|021\rangle \\
|120\rangle \\
|112\rangle
\end{array}\right.
$$

3. After Alice has encoded her bit on the travel qubit and she returns it, Eve applies the operation $Q_{t x y}^{-1}$ on the travel qubit and forwards it to Bob.

Eve then obtains some information about Alice's encoding by measuring her probes. To see how the attack works, we note that after Bob has received back the attacked travel qubit, the final state of the Alice-Bob-Eve system is

$$
\left|\psi^{a}\right\rangle_{h t x y}=\frac{1}{\sqrt{2}}(|012 a\rangle+|1020\rangle) .
$$


From this, one finds that the only non-vanishing probabilities $P_{A E B}$ are

$$
\begin{aligned}
& P_{000}=\frac{1}{2} \\
& P_{100}=P_{101}=P_{110}=P_{111}=\frac{1}{8} .
\end{aligned}
$$

This corresponds to a QBER of $\sum_{e}\left(p_{0 e 1}+p_{1 e 0}\right)=\frac{1}{4}$. Using these, one may compute the mutual information between Alice and Bob, $I_{A B} \equiv H(A)-H(A \mid B)$, where $H(A)$ and $H(A \mid B)$ are the classical (Shannon) entropy associated with probability distribution $P(a)$ and the conditional probability distribution $P(a \mid b)$ [58]. This is a measure of entropic correlation between Alice and Bob. Similarly, one defines the mutual information between Alice and Eve, given by $I_{A E} \equiv H(A)-H(A \mid E)$. From (3), one then finds that [44]

$$
I_{A B}=I_{A E}=\frac{3}{4} \log _{2} \frac{4}{3} \approx 0.311 .
$$

Thus, the attack makes the protocol insecure, since security (with one-way communication) requires that $I_{A B}>$ $I_{A E}$.

This attack is not symmetric between $a=0$ and $a=$ 1, and [44] proposes another, symmetric attack. Ref. [57] discusses a number of other attacks on the Pingpong protocol, showing it to be effectively robust against them. Thus, while the attack described is not known to be optimal, it represents a powerful and well-studied attack, and its performance under decoherence is likely to carry general implications of a wider nature, in particular the occurence of the "trusted noise" scenario. Therefore, our present work is focused on studying this aspect of it.

Furthermore, it is generally difficult to prove the security of a given QKD protocol against the most general (collective) attacks, though, specific protocols can be proposed where such security can be proven. Under the circumstances, a reasonable approach is to prove security against a non-general, but sufficiently powerful and sophisticated attack, which is the case here.

\section{QUANTUM COMMUNICATION UNDER A NOISY ENVIRONMENT}

The action of noise manifesting as a completely positive (CP) map on a system's density operator, can be given a Kraus representation:

$$
\phi(\rho)=\sum_{i} A_{i} \rho A_{i}^{\dagger}
$$

where the $A_{i}^{\prime} s$ must conform to the completeness constraint $\sum_{i} A_{i}^{\dagger} A_{i}=I$. In this work, we choose the simplified noise scenario depicted in Figure 1. In the onward leg, the noise first acts on the travel qubit, followed by Eve's attack $Q$ on this qubit, and then by Alice's encoding operation. In the return leg, this sequence is timereversed, so that Eve's second attack $Q^{-1}$ is followed by

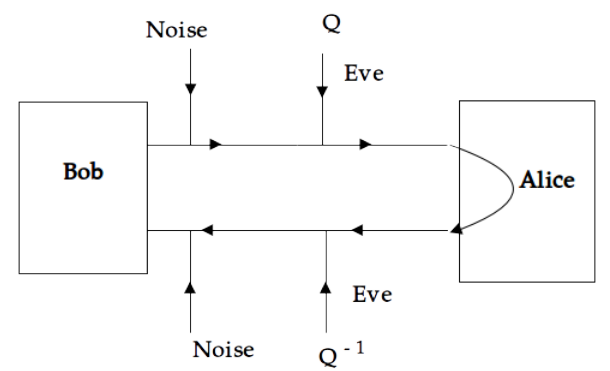

FIG. 1. Scenario of noise and attack as used in this work: Bob transmits to Alice one half of a Bell state, on which Alice encodes her bit by applying either the operation $I$ or $\sigma_{Z}$, before returning it to Bob. The action of noise is idealized as acting before Eve's action $Q$ in the onward leg and after her action $Q^{-1}$ in the return leg.

the noise, before receipt of the travel qubit and decoding of the two-qubit state by Bob.

In a noisy channel, suppose bits 0 and 1 correspond to noisy states $\rho^{a=0}$ and $\rho^{a=1}$. Then, the mutual information between the Alice and Bob is upper-bounded by the Holevo bound:

$$
\chi=S\left(\frac{\rho_{\mathrm{ht}}^{a=0}+\rho_{\mathrm{ht}}^{a=1}}{2}\right)-\frac{1}{2}\left[S\left(\rho_{\mathrm{ht}}^{a=0}\right)+S\left(\rho_{\mathrm{ht}}^{a=1}\right)\right],
$$

where $S(\rho) \equiv-\operatorname{Tr}[\rho \log (\rho)]$ denotes the von Neumann entropy.

We next consider noisy conditions with Eve's above attack on the Ping-Pong QKD protocol, with the travel qubit subjected to the amplitude damping (AD) [59] and depolarizing channels.

\section{A. Amplitude-Damping Noise}

The Kraus operators for AD channel are [28]:

$$
E_{0}^{A}=\left[\begin{array}{cc}
1 & 0 \\
0 & \sqrt{1-p}
\end{array}\right] ; E_{1}^{A}=\left[\begin{array}{cc}
0 & \sqrt{p} \\
0 & 0
\end{array}\right],
$$

where $p$ is the noise parameter, sometimes called the decoherence rate, and $0 \leq p \leq 1$.

The first attack of [44] (during the onward leg) makes the channel lossy and involves creating the vacuum state of the travel photon. This necessitates extending the qubit noise model (7) to that of a qutrit. There is no unique way to do this.

We use the extension represented by the following Kraus operators:

$$
E_{0}^{A}=\left[\begin{array}{ccc}
1 & 0 & 0 \\
0 & \sqrt{1-p} & 0 \\
0 & 0 & 1
\end{array}\right] ; E_{1}^{A}=\left[\begin{array}{ccc}
0 & \sqrt{p} & 0 \\
0 & 0 & 0 \\
0 & 0 & 0
\end{array}\right],
$$

which essentially implements the AD noise Eq. (7) on the polarization Hilbert space and does nothing to the 


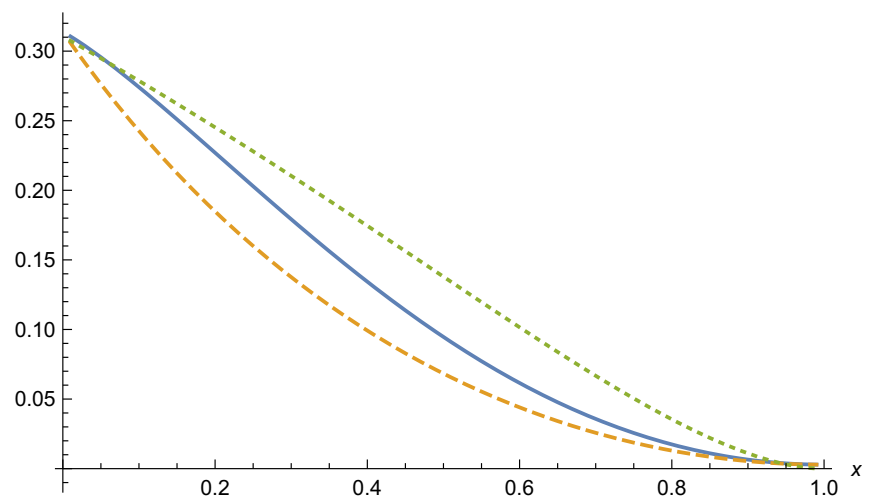

FIG. 2. (Color online) Performance of the modified Pingpong protocol under AD noise: The bold (blue), dashed (red) and dotted (green) plots represents $I_{A B}, I_{A E}$ and the Holevo bound for Alice-Bob. That $I_{A B}>I_{A E}$ for $0<p \leq 1$ implies that noise is beneficial to the legitimate users. In the noiseless limit, the Holevo bound coincides with $I_{A B}$, implying that the measurement strategy is optimal.

vacuum state. Here the vacuum state is taken to be the third dimension, denoted $|2\rangle$.

When the photon returns back to Bob, the state of the system hty for either encoding ' $a$ ' can be shown to have support of dimensionality 4 , spanned by the states $|010\rangle,|100\rangle,|011\rangle$ and $|000\rangle$, with the state of the $x$ particle being $|2\rangle$, as in the noiseless attack case. The final states with Bob-Eve for the encodings $a=0$ and $a=1$ are:

$$
\begin{aligned}
& \rho_{\text {hty }}^{a=0}=\frac{1}{2}\left(\begin{array}{cccc}
(1-p)^{2} & 1-p & 0 & 0 \\
1-p & 1 & 0 & 0 \\
0 & 0 & p(2-p) & 0 \\
0 & 0 & 0 & 0
\end{array}\right) ; \\
& \rho_{\text {hty }}^{a=1}=\frac{1}{2}\left(\begin{array}{cccc}
0 & 0 & 0 & 0 \\
0 & 1 & 0 & 1-p \\
0 & 0 & p(2-p) & 0 \\
0 & 1-p & 0 & (1-p)^{2}
\end{array}\right) .
\end{aligned}
$$

From Eq. (9), we obtain the following joint probabilities $p_{A E B}$, in place of Eq. (3):

$$
\begin{aligned}
& P_{000}=\frac{1}{8}(2-p)^{2} \\
& P_{001}=\frac{p^{2}}{8} \\
& P_{002}=P_{003}=P_{102}=P_{103}=\frac{1}{8}(2-p) p \\
& P_{110}=P_{111}=\frac{1}{8}(1-p)^{2} \\
& P_{010}=P_{011}=P_{012}=P_{013}=0 \\
& P_{100}=P_{101}=\frac{1}{8},
\end{aligned}
$$

with all other joint probability terms vanishing. Note that in the presence of AD noise, Bob will also obtain outcomes $\left|\phi^{ \pm}\right\rangle=\frac{1}{\sqrt{2}}(|00\rangle \pm|11\rangle)$ in his Bell state measurement, which corresponds to the outcome symbols 2 and 3 in Eq. (10).

From the above probabilities $P_{A E B}$, one derives the mutual information between Alice and Bob and that between Alice and Eve, to be

$$
\begin{aligned}
I(A: B) & =\frac{1}{8}\left[p \left(p \log \left(\frac{p^{2}}{2 p^{2}-2 p+2}\right)+p \log \left(\frac{8(p-2)^{2}}{(p-3) p+3}\right)+(p-2) \log \left(\frac{(p-2) p+2}{(p-1) p+1}\right)\right.\right. \\
& \left.+(p-2) \log \left(\frac{p-1}{(p-3) p+3}+1\right)\right)-2 p(p+2) \log (2)-4(p-1) \log \left(\frac{(p-2)^{2}}{2((p-3) p+3)}\right) \\
& \left.+2 \log \left(\frac{(p-2) p+2}{(p-1) p+1}\right)+2 \log \left(\frac{p-1}{(p-3) p+3}+1\right)+4\right],
\end{aligned}
$$

and

$$
\begin{aligned}
I(A: E) & =\frac{1}{8}\left(6+2 \log \left(\frac{1}{-p^{2}+2 p+3}\right)\right. \\
& \left.+(1-(p-2) p) \log \left(\frac{(p-2) p-1}{(p-3)(p+1)}\right)\right)
\end{aligned}
$$

respectively. These two quantities are depicted as a func- tion of noise $p$ in Figure 2. This shows that under the $\mathrm{AD}$ channel, there is a positive key rate $\kappa \equiv I_{A B}-I_{A E}$ for finite noise. It is as if the symmetry existing between Bob and Eve in terms of information gained, is broken by the noise, to the advantage of Alice and Bob. This is a surprising result, and implies that Alice and Bob will find this type of noise beneficial in this eavesdropping 
scenario.

If Alice and Bob are employing the original Ping-pong strategy and the eavesdropper is known to employ the above attack, then in the noise range $0<p<1$, Alice and Bob know that they can extract a finite secret key, after suitable privacy amplification.

From Eq. (9) one obtains the reduced density operators for the particles $h t$ :

$$
\begin{aligned}
& \rho_{h t}^{a=0}=\frac{1}{2}\left(\begin{array}{ccc}
(1-p)^{2} & 1-p & 0 \\
1-p & 1 & 0 \\
0 & 0 & p(2-p)
\end{array}\right) ; \\
& \rho_{h t}^{a=1} \quad=\frac{1}{2}\left(\begin{array}{ccc}
(1-p)^{2} & 0 & 0 \\
0 & 1 & 0 \\
0 & 0 & p(2-p)
\end{array}\right) .
\end{aligned}
$$

in the basis $\{|01\rangle,|10\rangle,|00\rangle\}$.

The maximum information Bob can receive is upperbounded by the Holevo quantity (6). To obtain this, we note that the eigenvalues $\lambda_{j}^{0}, \lambda_{j}^{1}$ and $\lambda_{j}^{01}$ for the density operators $\rho_{h t}^{a=0}, \rho_{h t}^{a=1}$ and their equal average, are:

$$
\begin{aligned}
\lambda_{j}^{0} & =\left\{0,-\frac{1}{2}(p-2) p, \frac{1}{2}((p-2) p+2)\right\} \\
\lambda_{j}^{1} & =\left\{\frac{1}{2}, \frac{1}{2}(p-1)^{2},-\frac{1}{2}(p-2) p\right\} \\
\lambda_{j}^{01} & =\left\{\frac{(2-p)}{2} p, \frac{1}{4}\left((p-2) p \pm \sqrt{(p-2) p(p-1)^{2}+1}+2\right)\right\}
\end{aligned}
$$

The Holevo bound (6) is thus given by:

$$
\chi_{A D}=h\left[\lambda_{j}^{01}\right]-\frac{1}{2}\left(h\left[\lambda_{j}^{0}\right]+h\left[\lambda_{j}^{1}\right]\right),
$$

where $h\left[\lambda_{j}^{\alpha}\right]=-\sum_{j=0}^{2} \lambda_{j}^{\alpha} \log _{2}\left(\lambda_{j}^{\alpha}\right)$. The quantity $\chi_{A D}$ is plotted in Figure 2 .

That the Holevo bound exceeding $I_{A B}$ here suggests that Bob's Bell state measurement strategy, although guaranteeing a positive key rate, is sub-optimal. Note that it is indeed optimal in the noiseless case.

\section{B. Depolarizing noise}

Consider the travel qubit subjected to depolarizing noise. This noise is characterized by the transformation $\rho \longrightarrow p \frac{\mathbb{I}}{2}+(1-p) \rho[30,60]$, for which the Kraus operators are:

$$
\begin{aligned}
& D_{0}=\sqrt{1-p}\left(\begin{array}{ll}
1 & 0 \\
0 & 1
\end{array}\right), D_{1}=\sqrt{\frac{p}{3}}\left(\begin{array}{cc}
0 & 1 \\
1 & 0
\end{array}\right) ; \\
& D_{2}=\sqrt{\frac{p}{3}}\left(\begin{array}{cc}
0 & -i \\
i & 0
\end{array}\right), D_{3}=\sqrt{\frac{p}{3}}\left(\begin{array}{cc}
1 & 0 \\
0 & -1
\end{array}\right),
\end{aligned}
$$

where $p=\left(1-\exp ^{-\frac{\tau t}{2}}\right), \tau$ being the decay factor. Here we shall use the extension of Eq. (16) given by:

$$
\begin{aligned}
& D_{0}=\sqrt{1-p}\left(\begin{array}{ccc}
1 & 0 & 0 \\
0 & 1 & 0 \\
0 & 0 & 1
\end{array}\right), D_{1}=\sqrt{\frac{p}{3}}\left(\begin{array}{ccc}
0 & 1 & 0 \\
1 & 0 & 0 \\
0 & 0 & 1
\end{array}\right) \\
& D_{2}=\sqrt{\frac{p}{3}}\left(\begin{array}{ccc}
0 & -i & 0 \\
i & 0 & 0 \\
0 & 0 & 1
\end{array}\right), D_{3}=\sqrt{\frac{p}{3}}\left(\begin{array}{ccc}
1 & 0 & 0 \\
0 & -1 & 0 \\
0 & 0 & 1
\end{array}\right)
\end{aligned}
$$

which essentially implements a depolarizing noise on the polarization Hilbert space and does nothing to the vacuum state.

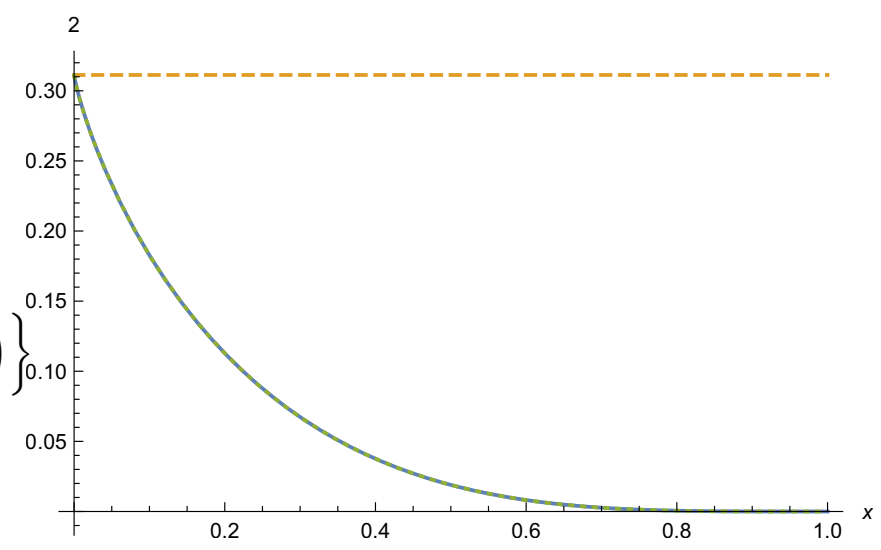

FIG. 3. (Color online) Performance of the Ping-pong QKD protocol under depolarizing noise: The bold (black) and dashed (red) plots represent $I_{A B}$ and $I_{A E}$ with the Holevo bound for Alice-Bob coinciding with $I_{A B}$. As a function of noise parameter $p, I_{A E}$ remains constant at the noiseless value of 0.311 , because Eve's attack strategy is indifferent to unital noise. That $I_{A B}$ equals the Holevo bound implies that Bob's Bell state measurement in the modified Ping-pong protocol is already optimal.

When the photon returns back to Bob, as per the scenario of Figure 1, with the noise given by the depolarizing channel, the state of the system hty for either encoding $a$ can be shown to have support of dimensionality 8 , spanned by the states $|j k l\rangle$, with $j, k, l \in\{0,1\}$, and the state of the $x$ particle being $|2\rangle$ as in the noiseless attack case. The final states with Bob-Eve for the encodings $a=0$ and $a=1$ are: 


$$
\begin{aligned}
& \rho^{0}=\frac{1}{2}\left(\begin{array}{cccccccc}
\frac{p(4-p)}{4} & 0 & 0 & 0 & 0 & 0 & 0 & 0 \\
0 & 0 & 0 & 0 & 0 & 0 & 0 & 0 \\
0 & 0 & \frac{(p-2)^{2}}{4} & 0 & (p-1)^{2} & 0 & 0 & 0 \\
0 & 0 & 0 & 0 & 0 & 0 & 0 & 0 \\
0 & 0 & (p-1)^{2} & 0 & \frac{(p-2)^{2}}{4} & 0 & 0 & 0 \\
0 & 0 & 0 & 0 & 0 & 0 & 0 & 0 \\
0 & 0 & 0 & 0 & 0 & 0 & \frac{p(4-p)}{4} & 0 \\
0 & 0 & 0 & 0 & 0 & 0 & 0 & 0
\end{array}\right) ; \\
& \rho^{1}=\frac{1}{2}\left(\begin{array}{cccccccc}
\frac{p(2-p)}{4} & 0 & 0 & 0 & 0 & 0 & 0 & 0 \\
0 & \frac{p(2-p)}{4} & 0 & 0 & 0 & 0 & 0 & 0 \\
0 & 0 & \frac{p^{2}}{4} & 0 & 0 & 0 & 0 & 0 \\
0 & 0 & 0 & \frac{(p-2)^{2}}{4} & (p-1)^{2} & 0 & 0 & 0 \\
0 & 0 & 0 & (p-1)^{2} & \frac{(p-2)^{2}}{4} & 0 & 0 & 0 \\
0 & 0 & 0 & 0 & 0 & \frac{p^{2}}{4} & 0 & 0 \\
0 & 0 & 0 & 0 & 0 & 0 & \frac{p(2-p)}{4} & 0 \\
0 & 0 & 0 & 0 & 0 & 0 & 0 & \frac{p(2-p)}{4}
\end{array}\right) .
\end{aligned}
$$

From Eq. (18), we obtain the following joint probabilities $P_{A E B}$, in place of Eq. (3):

$$
\begin{aligned}
& P_{000}=\frac{1}{2}+\frac{3 p}{8}(p-2) \\
& P_{001}=P_{002}=P_{003}=\frac{p}{8}(2-p) \\
& P_{010}=P_{011}=P_{012}=P_{013}=0 \\
& P_{100}=P_{101}=P_{110}=P_{111}=\frac{1}{8}+\frac{p}{16}(p-2) \\
& P_{102}=P_{103}=P_{112}=P_{113}=\frac{p}{16}(2-p)
\end{aligned}
$$

with all other joint probability terms vanishing. As with $\mathrm{AD}$ noise, here again Bob will also obtain outcomes $\left|\phi^{ \pm}\right\rangle=\frac{1}{\sqrt{2}}(|00\rangle \pm|11\rangle)$ in his Bell state measurement, which correspond to the outcome symbols 2 and 3 in Eq. (19).

From the above probabilities $P_{A E B}$, one finds the mutual information between Alice and Bob to be

$$
\begin{aligned}
I_{A B}(p) & =\frac{1}{36}\left[9 \log \left(\frac{4}{9} p(2 p-3)+1\right)\right. \\
& +8 p(2 p-3) \operatorname{coth}^{-1}\left(\frac{9}{(3-4 p)^{2}}\right) \\
& +6\left(4 p^{2}-6 p+3\right) \log \left(\frac{3}{4}-\frac{9}{64 p(2 p-3)+108}\right) \\
& \left.+(4 p(2 p-3)+9) \log \left(\frac{36}{16 p(2 p-3)+27}+4\right)\right] .
\end{aligned}
$$

On the other hand, it follows from Eq. (19) that

$$
\begin{aligned}
& P_{A E=00}=\frac{1}{2} ; P_{A E=01}=0 \\
& P_{A E=10}=P_{A E=11}=\frac{1}{4},
\end{aligned}
$$

i.e., $P_{A E}$ is independent of the noise parameter. Consequently, $I_{A E}(p)$ is just the noiseless value of $\frac{1}{8} \log \left(\frac{64}{27}\right)$.

Figure (3) shows that under the depolarizing channel, there is no positive key rate $\kappa \equiv I_{A B}-I_{A E}$ for finite noise, essentially because $I_{A E}$ remains constant, being unaffected by the depolarizing noise ( as explained above), whereas $I_{A B}$ drops with the noise level. Therefore, this channel, in contrast to the AD channel, offers no advantage to Alice and Bob in our scenario.

A similar disadvantageous behavior holds for dephasing and other unital noisy channels, which may be understood generally as follows. In our scenario, the noise acts before the first attack by Eve (see Figure 1), and the second instance of noise (in the backward trip of the particle) acts after Eve's second attack.

Therefore, the second instance of noise doesn't affect $I_{A E}$ (though, in general, it will affect $I_{A B}$ ). As to the onward trip of the particle, the travel qubit, as seen by Eve, is initially in a maximally mixed state $\frac{I}{2}$. Depolarizing noise or any other unital channel $\mathcal{C}_{U}$ is characterized by the property

$$
\mathcal{C}_{U}: \frac{I}{2} \mapsto \frac{I}{2},
$$

ie., it maps the state $\frac{I}{2}$ to itself. Thus, this state of the travel qubit remains unaffected, and hence Eve's correlation with Alice is indifferent to the noise.

It is worth noting here that if the unital noise acts after Eve's first intervention (rather than before, see Figure 1), 
then $I_{A E}$ is not expected to be invariant under the noise, since Eve's action can deviate the state of the particle from $\frac{I}{2}$.

From Eq. (18) one obtains the reduced density operators for the state of particles $h t$

$$
\rho_{h t}^{a=0}=\frac{1}{4}\left(\begin{array}{cccc}
(2-q) q & 0 & 0 & 0 \\
0 & ((q-2) q+2) & 2(q-1)^{2} & 0 \\
0 & 2(q-1)^{2} & (q-2) q+2 & 0 \\
0 & 0 & 0 & (2-q) q
\end{array}\right)
$$

As with Eq. (14), the maximum information Bob can receive is upper-bounded by the Holevo quantity (6). To derive this, we obtain the eigenvalues $\lambda_{j}^{0}, \lambda_{j}^{1}$ and $\lambda_{j}^{01}$ for the density operators $\rho_{h t}^{a=0}, \rho_{h t}^{a=1}$ and their equal average, which are found to be:

$$
\begin{aligned}
& \lambda_{j}^{0}=\frac{1}{4}\{(2-p) p,(2-p) p,(2-p) p, 3(p-2) p+4\}, \\
& \lambda_{j}^{1}=\frac{1}{4}\{(2-p) p,(2-p) p,(p-2) p+2,(p-2) p+2\} \\
& \lambda_{j}^{01}=\frac{1}{4}\{1,(2-p) p,(2-p) p, 2(p-2) p+3\} .
\end{aligned}
$$

Using this, the Holevo bound $\chi_{D P}$ under the depolarizing channel can be found in a manner similar to Eq. (15). Interestingly $\chi_{D P}$ is found to coincide with $I_{A B}$. This coincidence suggests that the Bell state measurement strategy by Bob is indeed optimal, unlike in the case of the AD channel.

\section{CONCLUSION AND DISCUSSIONS}

It is generally accepted that noise is detrimental to quantum information processing, in particular quantum cryptography. Here we identify, counter to this expectation, a scenario of "trusted noise", where noise can play a helpful role. In quantum key distribution, proofs of unconditional security assume that the eavesdropper Eve is restricted only by physical laws, and that all the noise is due to her attack. We consider a more realistic scenario, where Eve too is bound by limits imposed by noise due to environment-induced decoherence. We show how this can work to the advantage of legitimate parties, when noise affects the eavesdropper more than the legitimate parties. Now, an easy version of this scenario would have been one, where noise universally affects not just the legitimate parties, but also Eve. Therefore, the nontrivial aspect is that the noise only affects the communication channel and not the eavesdropping channel directly. Eve's limitation is her inability to replace the noisy communication channel between Alice and Bob by an noiseless one. In the particular situation considered here, the security of the Ping-Pong protocol (modified to a key distribution scheme) against a noise-restricted adversary is shown to improve under a non-unital decoherence, but to deteriorate under unital decoherence.
In light of [32-34], we may ask whether the AD statistics Eq. (10) can be produced using only local uncorrelated classical noise added by Alice and Bob, starting from the noiseless case Eq. (3). We now answer the question in the negative.

Alice's most general noise can be modelled by a combination of a conditional probability distribution $P^{A}(x \mid y)$ (used with probability $\alpha$ ) and a random coin toss $\varphi^{A}$ (used with probability $1-\alpha$ ), while that for Bob by a combination of a conditional probability distribution $P^{B}(x \mid y)$ (used with probability $\beta$ ) and a random coin toss $\varphi^{B}$ (used with probability $1-\beta$ ).

Further, let $P^{A}(0 \mid 0)=g, P^{A}(0 \mid 1)=h$ and $P^{B}(0 \mid 0)=$ $a, P^{B}(1 \mid 0)=b, P^{B}(2 \mid 0)=c, P^{B}(3 \mid 0)=1-a-b-c$; and $P^{B}(0 \mid 1)=d, P^{B}(1 \mid 1)=e, P^{B}(2 \mid 1)=f, P^{B}(3 \mid 1)=1-$ $d-e-f$. Applying the noise unilaterally on her side, Alice can't reproduce Eq. (10) because of the occurence of symbols 2 and 3 on Bob's side. Suppose Bob alone applies his local noise. Then, one finds that $P_{101}^{B}=P_{110}^{B}=$ $\frac{a+d}{8}$, which stands in contradiction with the data in Eq. (10). Thus, we must consider whether both Alice and Bob applying local noise independently can reproduce the required statistics. In the above, $P_{j}^{A}$ denotes the $j$ th component of the joint probability distribution obtained by Alice's application of her local classical noise to the classical outcome data of Eq. (3); analogously for $P_{j}^{B}$ in the case of Bob.

Without loss of generality, suppose Alice applies her local noise first, and then Bob. We shall use the notation where the $j$ th component after Bob also has applied his local classical noisy channel to the classical data $P_{j}^{A}$ is denoted $P_{j}^{A \rightarrow B}$. Then, from Eq. (3), we obtain:

$$
P_{010}^{A}=\frac{\alpha h}{8}+\frac{(1-\alpha) r}{8} .
$$

This must, in view of the vanishing of this component in Eq. (10), implying

$$
\begin{aligned}
\alpha=0, & r=0 \\
\alpha=1, & h=0 .
\end{aligned}
$$

If $P_{010}^{A}$ doesn't vanish, then we must have $p^{B}(0 \mid 0)=0$, to ensure that under the transformation induced by Bob's play, the final $P_{010}^{A \rightarrow B}$ vanishes. This would mean that $p^{B}(1 \mid 0)$ or $p^{B}(2 \mid 0)$ or $p^{B}(3 \mid 0)$ should be non-vanishing. But this, in turn, would mean that $P_{011}^{A \rightarrow B}$ or $P_{012}^{A \rightarrow B}$ or $P_{013}^{A \rightarrow B}$ should be non-vanishing, in contradiction with the corresponding requirement in data Eq. (10). Thus, we are led to conditions Eq. (24).

To see why condition Eq. (24a) won't work out, we note that it would imply that $P_{000}^{A}=\frac{\alpha}{2}\left(g+\frac{h}{4}\right)+(1-$ $\alpha) r \frac{5}{8} \equiv 0$ as well as $p_{001}^{A}=\frac{\alpha h}{8}+(1-\alpha) \frac{r}{8} \equiv 0$. But, this would imply that

$$
\begin{aligned}
P_{000}^{A \rightarrow B} & =\beta\left(a P_{000}^{A}+d P_{001}^{A}\right)+(1-\beta) q\left(p_{000}^{A}+P_{001}^{A}\right) \\
& =0
\end{aligned}
$$

contradicting the fact that this component is nonvanishing in the AD statistics Eq. (10). 
To see why condition Eq. (24b) also won't work out, we note that it would imply that

$$
\begin{aligned}
& P_{000}^{A \rightarrow B}=\frac{g}{2}(\beta a+(1-\beta) q), \\
& P_{100}^{A \rightarrow B}=\frac{g}{8}(\beta a+(1-\beta) q),
\end{aligned}
$$

implying that these two components differ by a factor 4 , contradicting the additional noise dependence seen in Eq. (10).

In conclusion, the advantage provided by the quantum AD channel can't be simulated locally (without any classical communication) by the legitimate parties, acting on the noiseless (but eavesdropped) outcome statistics. This may be attributed to the fundamentally quantum nature of the disturbance introduced into the noisy channel through Eve's intervention.

\section{ACKNOWLEDGEMENTS}

VS thanks the Ministery of Human Resource Development, Govt. of India, for offering a doctoral fellowship as a Ph.D. research scholar at Indian Institute of Technology Jodhpur, Rajasthan, India. SB thanks Atul Kumar and Anirban Pathak for useful discussions during the preliminary stage of this work. SB acknowledges support by the project number 03(1369)/16/EMR-II funded by Council of Scientific and Industrial Research, New Delhi, India. US and RS thank DST-SERB, Govt. of India, for financial support provided through the project EMR/2016/004019.
[1] Nicolas Gisin, Grégoire Ribordy, Wolfgang Tittel, and Hugo Zbinden. Quantum cryptography. Reviews of modern physics, 74(1):145, 2002.

[2] N Srinatha, S Omkar, R Srikanth, Subhashish Banerjee, and Anirban Pathak. The quantum cryptographic switch. Quantum information processing, pages 1-12, 2014.

[3] Vishal Sharma. Effect of noise on practical quantum communication systems. Defence Science Journal, 66(2):186192, 2016.

[4] Vishal Sharma, Chitra Shukla, Subhashish Banerjee, and Anirban Pathak. Controlled bidirectional remote state preparation in noisy environment: a generalized view. Quantum Information Processing, 14(9):34413464, 2015.

[5] Vishal Sharma, Kishore Thapliyal, Anirban Pathak, and Subhashish Banerjee. A comparative study of protocols for secure quantum communication under noisy environment: single-qubit-based protocols versus entangledstate-based protocols. Quantum Information Processing, 15(11):4681-4710, 2016.

[6] Vishal Sharma and Richa Sharma. Analysis of spread spectrum in matlab. International Journal of Scientific \& Engineering Research, 5(1), 2014.

[7] Gang Wang, Dan Shen, Genshe Chen, Khanh Pham, and Erik Blasch. Polarization tracking for quantum satellite communications. In Sensors and Systems for Space Applications VII, volume 9085, page 90850T. International Society for Optics and Photonics, 2014.

[8] Vishal Sharma and Subhashish Banerjee. Analysis of atmospheric effects on satellite based quantum communication: A comparative study. arXiv:1711.08281.

[9] Charles H Bennett and Gilles Brassard. Quantum cryptography: Public key distribution and con tos5. 1984.

[10] Artur K Ekert. Quantum cryptography based on bellâĂŹs theorem. Physical review letters, 67(6):661, 1991.

[11] Charles H Bennett. Quantum cryptography using any two nonorthogonal states. Physical review letters, 68(21):3121, 1992.

[12] Lior Goldenberg and Lev Vaidman. Quantum cryptography based on orthogonal states. Physical Review Letters, 75(7):1239, 1995.
[13] Hoi-Kwong Lo and Hoi Fung Chau. Unconditional security of quantum key distribution over arbitrarily long distances. science, 283(5410):2050-2056, 1999.

[14] Valerio Scarani and Nicolas Gisin. Quantum key distribution between $N$ partners: Optimal eavesdropping and bell's inequalities. Phys. Rev. A, 65:012311, Dec 2001.

[15] Hoi-Kwong Lo, Hoi Fung Chau, and Mohammed Ardehali. Efficient quantum key distribution scheme and a proof of its unconditional security. Journal of Cryptology, 18(2):133-165, 2005.

[16] Valerio Scarani, Helle Bechmann-Pasquinucci, Nicolas J Cerf, Miloslav Dušek, Norbert Lütkenhaus, and Momtchil Peev. The security of practical quantum key distribution. Reviews of modern physics, 81(3):1301, 2009.

[17] Anirban Pathak. Elements of quantum computation and quantum communication. Taylor \& Francis, 2013.

[18] Akshata Shenoy-Hejamadi, Anirban Pathak, and Srikanth Radhakrishna. Quantum cryptography: Key distribution and beyond. Quanta, 6(1):1-47, 2017.

[19] Fu-Guo Deng and Gui Lu Long. Secure direct communication with a quantum one-time pad. Phys. Rev. A, 69:052319, May 2004.

[20] Kim Boström and Timo Felbinger. Deterministic secure direct communication using entanglement. Physical Review Letters, 89(18):187902, 2002.

[21] Marco Lucamarini and Stefano Mancini. Secure deterministic communication without entanglement. Physical review letters, 94(14):140501, 2005.

[22] C. Shukla, A. Pathak, and R. Srikanth. Beyond the Goldenberg-Vaidman protocol: Secure and efficient quantum communication using arbitrary, orthogonal, multi-particle quantum states. Int. J. Quantum Inf., 10:1241009, 2012.

[23] Anna Pappa, André Chailloux, Eleni Diamanti, and Iordanis Kerenidis. Practical quantum coin flipping. Phys. Rev. A, 84:052305, Nov 2011.

[24] Ryan Amiri and Juan Miguel Arrazola. Quantum money with nearly optimal error tolerance. Physical Review A, 95(6):062334, 2017.

[25] Chun-Yan Wei, Xiao-Qiu Cai, Bin Liu, Tianyin Wang, and Fei Gao. A generic construction of quantum- 
oblivious-key-transfer-based private query with ideal database security and zero failure. IEEE Transactions on Computers, 67:2-8, 2017.

[26] Run-hua Shi, Yi Mu, Hong Zhong, Shun Zhang, and Jie Cui. Quantum private set intersection cardinality and its application to anonymous authentication. Information Sciences, 370:147-158, 2016.

[27] Subhashish Banerjee and R Srikanth. Geometric phase of a qubit interacting with a squeezed-thermal bath. The European Physical Journal D-Atomic, Molecular, Optical and Plasma Physics, 46(2):335-344, 2008.

[28] R Srikanth and Subhashish Banerjee. Squeezed generalized amplitude damping channel. Physical Review A, 77(1):012318, 2008.

[29] Subhashish Banerjee and R Ghosh. Dynamics of decoherence without dissipation in a squeezed thermal bath. Journal of Physics A: Mathematical and Theoretical, 40(45):13735, 2007.

[30] S Omkar, R Srikanth, and Subhashish Banerjee. Dissipative and non-dissipative single-qubit channels: dynamics and geometry. Quantum information processing, 12(12):3725-3744, 2013.

[31] Satyabrata Adhikari, Dipankar Home, Archan S Majumdar, Alok Kumar Pan, Akshata Shenoy, and R Srikanth. Toward secure communication using intra-particle entanglement. Quantum Information Processing, 14(4):14511468, 2015.

[32] Renato Renner, Nicolas Gisin, and Barbara Kraus. Information-theoretic security proof for quantum-keydistribution protocols. Phys. Rev. A, 72:012332, Jul 2005.

[33] Stefano Pirandola, Raul García-Patrón, Samuel L. Braunstein, and Seth Lloyd. Direct and reverse secretkey capacities of a quantum channel. Phys. Rev. Lett., 102:050503, Feb 2009.

[34] Raúl García-Patrón and Nicolas J. Cerf. Continuousvariable quantum key distribution protocols over noisy channels. Phys. Rev. Lett., 102:130501, Mar 2009.

[35] Subhashish Banerjee, R. Srikanth, C. M. Chandrashekar, and Pranaw Rungta. Symmetry-noise interplay in a quantum walk on an n-cycle. Phys. Rev. A, 78:052316, Nov 2008.

[36] Gui-lu Long, Fu-guo Deng, Chuan Wang, Xi-han Li, Kai Wen, and Wan-ying Wang. Quantum secure direct communication and deterministic secure quantum communication. Frontiers of Physics in China, 2(3):251-272, 2007.

[37] Chuan Wang, Fu-Guo Deng, Yan-Song Li, Xiao-Shu Liu, and Gui Lu Long. Quantum secure direct communication with high-dimension quantum superdense coding. Physical Review A, 71(4):044305, 2005.

[38] Fu-Guo Deng and Gui Lu Long. Secure direct communication with a quantum one-time pad. Physical Review A, 69(5):052319, 2004.

[39] Gao Ting, Yan Feng-Li, and Wang Zhi-Xi. A simultaneous quantum secure direct communication scheme between the central party and other $\mathrm{m}$ parties. Chinese Physics Letters, 22(10):2473, 2005.

[40] Chuan Wang, Fu Guo Deng, and Gui Lu Long. Multistep quantum secure direct communication using multiparticle green-horne-zeilinger state. Optics communications, 253(1):15-20, 2005.

[41] Xi-Han Li, Chun-Yan Li, Fu-Guo Deng, Ping Zhou, YuJie Liang, and Hong-Yu Zhou. Quantum secure direct communication with quantum encryption based on pure entangled states. arXiv preprint quant-ph/0512014, 2005.

[42] Xing-Ri Jin, Xin Ji, Ying-Qiao Zhang, Shou Zhang, SucKyoung Hong, Kyu-Hwang Yeon, and Chung-In Um. Three-party quantum secure direct communication based on ghz states. Physics Letters A, 354(1):67-70, 2006.

[43] Man Zhong-Xiao and Xia Yun-Jie. Improvement of security of three-party quantum secure direct communication based on ghz states. Chinese Physics Letters, 24(1):15, 2007.

[44] Antoni Wójcik. Eavesdropping on the ping-pong quantum communication protocol. Physical Review Letters, 90(15):157901, 2003.

[45] Yun-Guang Han, Zhen-Qiang Yin, Hong-Wei Li, Wei Chen, Shuang Wang, Guang-Can Guo, and Zheng-Fu Han. Security of modified ping-pong protocol in noisy and lossy channel. Scientific reports, 4:4936, 2014.

[46] Piotr Zawadzki. Security of ping-pong protocol based on pairs of completely entangled qudits. Quantum Information Processing, pages 1-12, 2012.

[47] Piotr Zawadzki. The ping-pong protocol with a prior privacy amplification. International Journal of Quantum Information, 10(03):1250032, 2012.

[48] Qing-Yu Cai and Bai-Wen Li. Improving the capacity of the boström-felbinger protocol. Physical Review A, 69(5):054301, 2004.

[49] Cai Qing-Yu and Li Bai-Wen. Deterministic secure communication without using entanglement. Chinese Physics Letters, 21(4):601, 2004.

[50] Qing-Yu Cai. Eavesdropping on the two-way quantum communication protocols with invisible photons. Physics Letters A, 351(1-2):23-25, 2006.

[51] Piotr Zawadzki and Jarosław Adam Miszczak. A general scheme for information interception in the ping-pong protocol. Advances in Mathematical Physics, 3162012:1-7, 2016.

[52] Jian Li, DJ Song, XJ Guo, and Bo JING. An improved security detection strategy based on w state in âĂIJpingpongâĂ̇ protocol. Chin J Electron, 21:117-120, 2012.

[53] Piotr Zawadzki. Improving security of the ping-pong protocol. Quantum Information Processing, 12(1):149-155, 2012.

[54] Zhanjun Zhang, Zhongxiao Man, and Yong Li. Improving wójcik's eavesdropping attack on the ping-pong protocol. Physics Letters A, 333(1):46-50, 2004.

[55] Eugene V Vasiliu. Non-coherent attack on the pingpong protocol with completely entangled pairs of qutrits. Quantum Information Processing, 10(2):189-202, 2011.

[56] Arti Chamoli and CM Bhandari. Secure direct communication based on ping-pong protocol. Quantum Information Processing, 8(4):347-356, 2009.

[57] Kim Boström and Timo Felbinger. On the security of the ping-pong protocol. Physics Letters A, 372(22):39533956, 2008.

[58] Michael A Nielsen and Isaac L Chuang. Quantum computation and quantum information. Cambridge university press, 2010.

[59] R Srikanth and Subhashish Banerjee. An environmentmediated quantum deleter. Physics Letters A, 367(4):295-299, 2007.

[60] Michael A Nielsen and Isaac L Chuang. Quantum computation and quantum information. Quantum, 546:1231, 2000 . 